\title{
High neural precursor cell-expressed developmentally down-regulated 9 expression is a poor prognostic indicator for osteosarcoma
}

\author{
Jinyi Feng ${ }^{1 *}$, Yang Gu ${ }^{2 \#}$, Linquan $\mathrm{Zhou}^{2}$, Gang Rui ${ }^{1}$, Wenge Liu ${ }^{2}$ \\ ${ }^{1}$ Department of Orthopedic Surgery, The First Affiliated Hospital of Xiamen University, Xiamen, China; ${ }^{2}$ Department of Orthopedic Surgery, \\ Affiliated Union Hospital of Fujian Medical University, Fuzhou, China \\ Contributions: (I) Conception and design: J Feng, Y Gu; (II) Administrative support: W Liu, G Rui; (III) Provision of study materials or patients: \\ J Feng, Y Gu, L Zhou; (IV) Collection and assembly of data: J Feng, Y Gu, L Zhou; (V) Data analysis and interpretation: Y Gu; (VI) Manuscript \\ writing: All authors; (VII) Final approval of manuscript: All authors. \\ \#These authors contributed equally to this work. \\ Correspondence to: Wen-Ge Liu, PhD, MD. Department of Orthopedics Surgery, Affiliated Union Hospital of Fujian Medical University, 29 Xinquan \\ Road, Gulou District, Fuzhou, China. Email: 13705977551@163.com; Gang Rui, PhD, MD. Department of Orthopedics Surgery, The First \\ Affiliated Hospital of Xiamen University, 55 Zhenhai Road, Siming District, Xiamen, China. Email: reigang@163.com.
}

Background: This study detected neural precursor cell-expressed developmentally down-regulated 9 (NEDD9) expression in osteosarcoma tissue samples. It also examined the association between NEDD9 expression and clinicopathological features of osteosarcoma.

Methods: The prognostic value of NEDD9 has been verified in multiple solid cancers, but not osteosarcoma. In this study, 45 osteosarcoma paraffin-embedded specimens were accumulated and applied based on immunohistochemistry to identify NEDD9 expression. A correlation linking NEDD9 expression, clinicopathological qualities, and its prognostic significance was assessed to verify whether NEDD9 could be a poor prognosis biomarker.

Results: High NEDD9 expression compared to its low expression in osteosarcoma tissue was shown in the immunohistochemical assay. The percentage of high and low NEDD9 expression was $62.2 \%$ and $37.8 \%$ respectively. NEDD9 expression level was significantly associated with metastasis and Enneking stage $(\mathrm{P}<0.05)$, while gender, age, tumor size, sites, and histopathological types were not. Univariate analysis showed that metastasis with a hazard ratio $(\mathrm{HR})$ of $2.63(\mathrm{P}=0.003)$, Enneking stage III $(\mathrm{HR}=5.44, \mathrm{P}=0.009)$, chondroblastic osteosarcoma ( $\mathrm{HR}=2.59, \mathrm{P}=0.014)$, and high NEDD9 expression $(\mathrm{HR}=2.79, \mathrm{P}=0.002$ ) were prognostic factors contributing to poor overall survival (OS). Moreover, multivariate analysis revealed that nonconventional pathological osteosarcoma ( $\mathrm{HR}=0.17, \mathrm{P}=0.010)$ and NEDD9 expression $(\mathrm{HR}=6.03$, $\mathrm{P}<0.001)$ were independent prognostic factors.

Conclusions: High NEDD9 expression in osteosarcoma independently indicated poor clinical prognosis, indicating the possibility that NEDD9 could be a therapeutic target for osteosarcoma.

Keywords: Neural precursor cell-expressed developmentally down-regulated 9 (NEDD9); osteosarcoma; immunohistochemistry; prognosis

Submitted Mar 05, 2020. Accepted for publication Sep 12, 2020.

doi: $10.21037 /$ tcr-20-1354a

View this article at: http://dx.doi.org/10.21037/tcr-20-1354a 


\section{Introduction}

Osteosarcoma is the most typically observed and dangerous bone cancer that occurs primarily among young adults and has a poor survival rate. It comprises about $0.2 \%$ of malignant tumors. Its development and progression are complex processes involving numerous genes, so its underlying mechanisms remain unclear $(1,2)$. Therefore, it is essential to recognize the key molecular markers influencing its prognosis to potentially develop a specific targeted drug therapy (3).

Neural precursor cell-expressed developmentally downregulated 9 (NEDD9), or human enhancer of filamentation 1 (HEF1) and Crk-associated substrate lymphocyte type (Cas-L), is a Crk-associated substrate. A meta-analysis has documented that NEDD9 overexpression is associated with poor prognosis in multiple solid cancers, with a hazard ratio (HR) of 1.82 (95\% CI: 1.43-2.31) (4). NEDD9 is essential for activating cell migration in integrin-dependent signaling cascades, and laterally communicates with Ras signaling cascades (5). Moreover, NEDD9 interacts with FAK-SrcPI3K signaling cascades to initiate cell proliferation and invasion (6). NEDD9 is also correlated with the TGFbeta signaling pathway regulating tissue remodeling and epithelial-mesenchymal transition (EMT), and promotes cancer metastasis (7).

NEDD9 expression has been linked to brain, head, neck, ovary, colon, stomach, breast, pancreas, kidney, lung, and liver cancer (4). However, no similar research exists on the relationship between NEDD9 expression and osteosarcoma prognosis. In the present study, NEDD9 expression in osteosarcoma tissue was examined and the influence of its expression on overall survival (OS) in osteosarcoma patients was evaluated, which would help identify whether NEDD9 could be a prognosis-predictive marker for osteosarcoma.

We present the following article in accordance with the REMARK reporting checklist (available at http://dx.doi. org/10.21037/tcr-20-1354a).

\section{Methods}

\section{Patients and specimen qualities}

This study enrolled 45 cases of primary untreated osteosarcoma between January 2008 and December 2014. All osteosarcoma patients were clearly diagnosed by a senior pathologist after puncture or incision biopsy specimens. The preoperative neoadjuvant chemotherapy with methotrexate, cisplatin, and doxorubicin were operated for two courses for all patients. The same senior doctors performed these patients' treatments. Preoperative chemotherapy was used as a basis for conducting postoperative chemotherapy. Patients not undergoing surgical treatment or who had a poor response to the neoadjuvant chemotherapy were excluded. The study was conducted in accordance with the Declaration of Helsinki (as revised in 2013). The study was approved by the Ethics Committees of the First Affiliated Hospital of Xiamen University (No.2019KY019) and all patients signed an informed consent form. Specimens were obtained from archived and paraffin-embedded surgical sample tissue sections from 45 osteosarcoma patients from the pathological specimen bank at FAHXMU.

\section{Immunohistochemistry (IHC) for NEDD9 and evaluation}

The formalin-fixed and paraffin-embedded tumor samples were performed with IHC staining, which was used to detect NEDD9 based on the streptavidin-peroxidase complex method (8). Osteosarcoma tissue obtained from every case was fixed using formalin and processed with paraffin wax-embedded tissue blocks. Sections were cut onto glass slides, xylene was used to deparaffinize them, and a graded series of ethanol was used to rehydrate them. These sections were incubated in citrate buffer and 3\% hydrogen peroxide for antigen retrieval and endogenous peroxidase blocking. Nonspecific binding of antibodies was blocked by incubating the sections with $5 \%$ bovine serum albumin (BSA) and the sections were washed. Rabbit polyclonal antibody was used to incubate these sections against human NEDD9 (1:100 dilution; cat no. PA5-102624, Thermo Fisher Scientific, Waltham, MA, USA) during the night at $4{ }^{\circ} \mathrm{C}$, followed by an hour of room-temperature incubation using horseradish peroxidase-conjugated secondary goat anti-rabbit antibody (1:50 dilution; cat no. A0208, Beyotime Institute of Biotechnology, Shanghai, China). Phosphate buffered saline (PBS) was then used to wash the sections and a metal enhanced 3,3'-diaminobenzidine (DAB) solution (cat no. P0202, Beyotime Institute of Biotechnology, Shanghai, China) was used to visualize the antigen-antibody complex. The immunostaining degree was determined and scored independently by two observers who were blinded to the clinical data and scored independently based on the intensity of staining and percentage of NEDD9 immunoreactive cells. Each section's percentage of stained tumor cells was counted and scored: $0-5 \%$ staining as $0,6-25 \%$ of cells stained as $1,25-50 \%$ as 2 , and $>51 \%$ as 3 . The scores for NEDD9 staining intensity were assessed: 0 (no staining), 1 
(weak), 2 (moderate), and 3 (strong). Each case's score was multiplied with results of $0,1,2,3,4,6$, or 9. Low NEDD9 expression scores were $0-3$ and high expression scores were 4-9 $(9,10)$.

\section{Clinicopatbological features and follow-up}

This study was single-center retrospective and all clinicopathological features were collected, including gender, age, tumor size, metastasis, Enneking stage, tumor sites, and histopathological types. It had a small sample size due to the low incidence of osteosarcoma and exclusion criteria.

After medical records were reviewed and phone calls were used to contact the patients or their relatives, five years of follow-up data was found that dated to December 31,2019 . OS was calculated according to the time between surgery and death (or the latest follow-up).

\section{Statistical analysis}

IBM SPSS Statistics, version 23.0 (IBM Corp., Armonk, NY, USA) was used to conduct statistical analysis. Pearson's chi-square or Fisher's exact tests were used to compare clinicopathological factors among groups. The KaplanMeier method and log-rank tests were used during survival curve analysis and for determining statistical differences in GraphPad Prism 7.0 (GraphPad Software Inc., San Diego, CA, USA). A Cox proportional-hazards regression model was used to analyze univariate and multivariate survival. Baseline variables that showed a univariate relationship with an outcome were entered into this regression model. Statistical significance was noted in $\mathrm{P}$ values $<0.05$ for these tests.

\section{Results}

\section{Patient qualities}

The study cohort consisted of 26 male and 19 female osteosarcoma patients. Patients under 20 years old accounted for $75.6 \%$ of all cases in the whole cohort. Furthermore, 23 (51.1\%), 13 (28.9\%), and 9 (20.0\%) tumors were in the femur, tibia, and other locations, respectively. Over half of the patients $(57.8 \%)$ had tumors $<8 \mathrm{~cm}$ in diameter. The Enneking stage included stage I (4 patients, $8.9 \%)$, stage II $(21,46.7 \%)$, and stage III (20, $44.4 \%)$, respectively. Based on histopathological type, 22 (48.9\%), 13 (28.9\%), 6 (13.3\%), and 4 (8.9\%) tumors were osteoblastic, chondroblastic, fibroblastic, and other types respectively.

\section{Association between NEDD9 level and clinical-pathological findings}

Typical NEDD9 staining in osteosarcoma tissue was presented in Figure 1. It was brown and yellow to dark yellow, and was grainy, diffused, or widespread. The percentage of tumors indicating high and low NEDD9 expression was $62.2 \%$ and $37.8 \%$ respectively. Table 1 indicated that the NEDD9 expression level had a significant connection with metastasis and the Enneking stage $(\mathrm{P}<0.05)$, while gender, age, tumor size, sites, and histopathological types lacked this connection.

\section{OS of osteosarcoma}

The average follow-up period was 34.9 months (range of 6-96 months). According to Figure 2A, the study involved Kaplan-Meier analyses that indicated unfavorable OS in patients having high NEDD9 expression compared to that in patients having low NEDD9 expression. Moreover, Figure 2B,C,D demonstrated that metastasis as well as Enneking stage had a significant relation to patient OS. It showed that the high NEDD9 expression, metastasis and increasing Enneking stage were associated with low survival rates $(\mathrm{P}=0.001)$. Although the $\mathrm{P}$ value was 0.065 , chondroblastic osteosarcoma in Figure $2 C$ showed the poorest prognosis compared with other pathological types, which resembled previously reported findings $(11,12)$.

Clinicopathological features affecting the prognosis of osteosarcoma patients were identified by using univariate and multivariate analysis. Table 2 indicates that univariate analysis demonstrated metastasis $(\mathrm{HR}=2.63, \mathrm{P}=0.003)$, Enneking stage III ( $\mathrm{HR}=5.44, \mathrm{P}=0.009)$, chondroblastic osteosarcoma $(\mathrm{HR}=2.59, \mathrm{P}=0.014)$, and high NEDD9 expression $(\mathrm{HR}=2.79, \mathrm{P}=0.002)$ as prognostic factors leading to poor OS. In addition, the multivariate analysis indicated the status of nonconventional pathological osteosarcoma ( $\mathrm{HR}=0.17, \mathrm{P}=0.010)$ and NEDD9 expression ( $\mathrm{HR}=6.03, \mathrm{P}<0.001)$ as independent prognostic factors.

\section{Discussion}

Osteosarcoma is the most typically observed and dangerous bone cancer that occurs primarily among young adults. Extensive treatment has produced limited results related to 
$100 x$
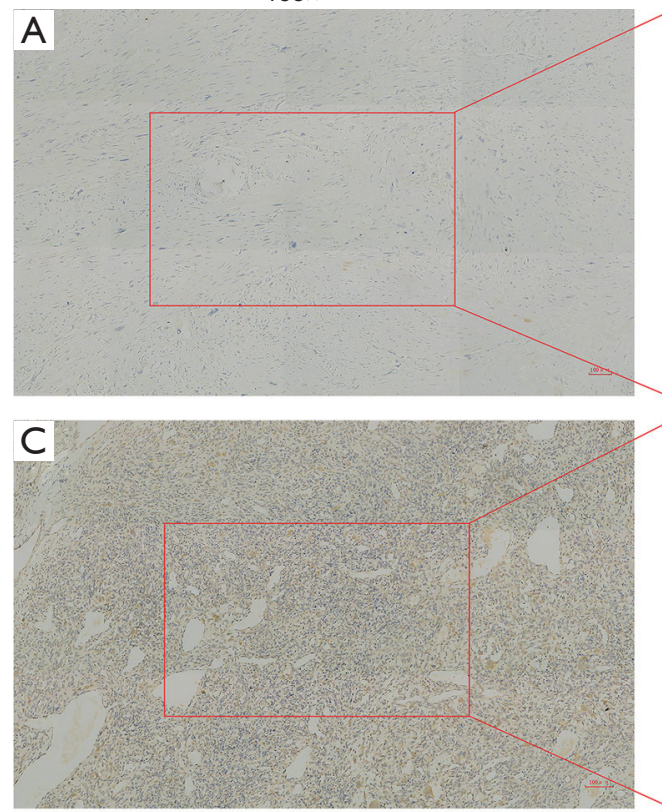

E

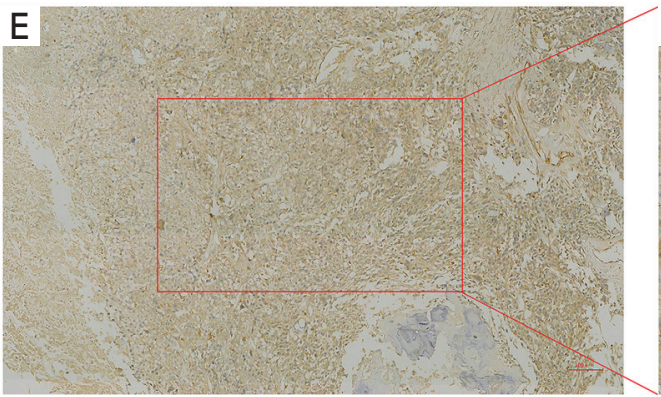

$200 x$
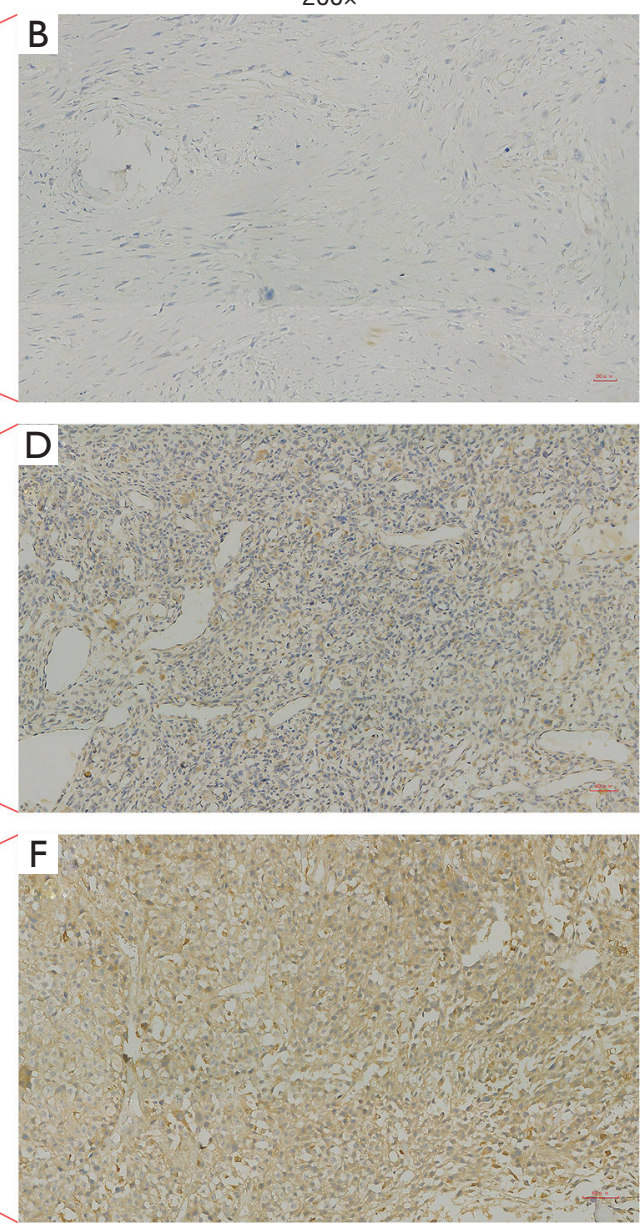

Figure 1 Immunohistochemistry (IHC) for neural precursor cell-expressed developmentally down-regulated 9 (NEDD9) in osteosarcoma. Osteosarcoma tissue was stained with an anti-NEDD9 antibody as negative staining (A,B). Low-level staining in osteosarcoma tissue (C,D). High-level staining in osteosarcoma tissue (E,F).

enhancing its prognosis (13). Thus, the focus should be on researching potential biomarkers during the progression of this cancer.

Modern achievements in tumor markers tend to emphasize NEDD9 and its role, established as a premetastatic marker for multiple tumor types (14). The relationship between NEDD9 expression and prognosis in esophageal cancer is controversial in lung cancer (15-17). However, 17 metaanalyzed peer-review articles and 2,393 individuals having solid cancers including breast, gastric, and ovarian cancer mentioned summarized statistics showing the negative effect of excessive NEDD9 expression on OS (4).

NEDD9 signaling supports invasion, survival, ciliary resorption, and mitosis (18). A lack of NEDD9 delays tumor growth rate and reduces the expression and activation of signaling proteins such as SRC, STAT3, E-cadherin, and AURKA (14). NEDD9 has been shown to regulate MICAL1, which enables Rac1-dependent and hypoxiainduced gastric cancer cell migration (19). Furthermore, it has an essential role during integrin-dependent signaling cascades that initiate cell migration, and facilitates lateral communication with Ras signaling cascades (5). Activated MMP14, MMP2, and MMP9 metalloproteinases result in invasion occurring along with ECM proteolysis (20).

In the present study, NEDD9 demonstrated extensive expression among samples of osteosarcoma tissue, and high expression was noted in $62.2 \%$ of them. This resembled a recent study mentioning that NEDD9 expression was significantly higher in 26 pairs of osteosarcoma tissues compared with matched normal tissues (21). Moreover, 
Table 1 The correlation between NEDD9 expression and the clinicopathological features

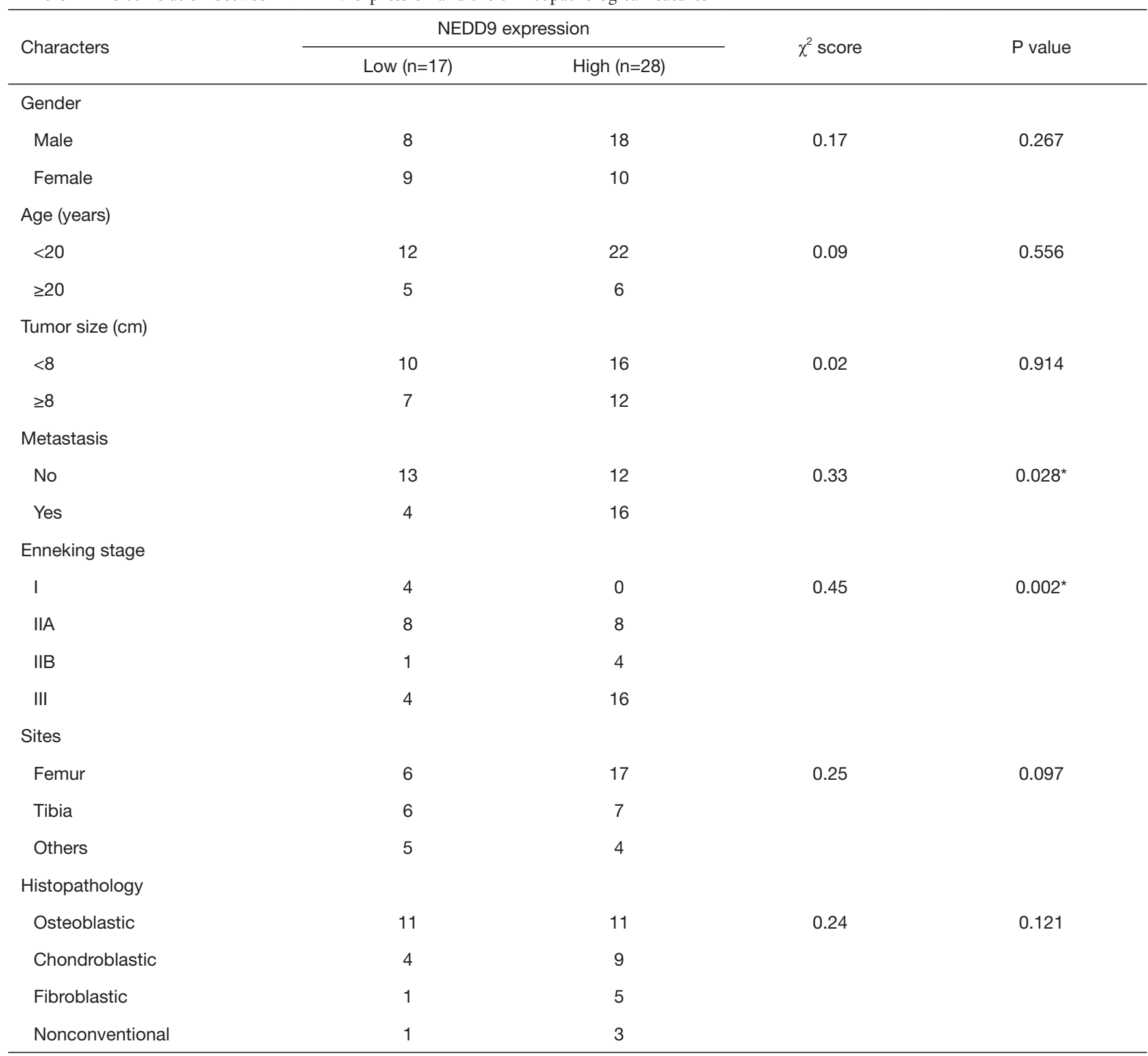

*, $\mathrm{P}<0.05$ was considered significant. NEDD9, neural precursor cell-expressed developmentally down-regulated 9.

the prognostic role of NEDD9 expression in osteosarcoma was indicated with IHC and demonstrated for the first time that high-level NEDD9 expression was correlated with clinicopathologic factors such as advanced Enneking stage and metastasis. Furthermore, Kaplan-Meier survival analyses indicated that osteosarcoma patients exhibiting high NEDD9 expression had more unfavorable clinical outcomes compared to those in patients exhibiting low
NEDD9 expression. This study's outcome produced further examples reconfirming the argument of high NEDD9 expression indicating that those with osteosarcoma will exhibit negative clinical results.

The development of new chemotherapeutic agents and targeted cancer therapy relies on research related to effective diagnostic and prognostic biomarkers. However, no satisfactory prognostic biomarkers have been found 
A

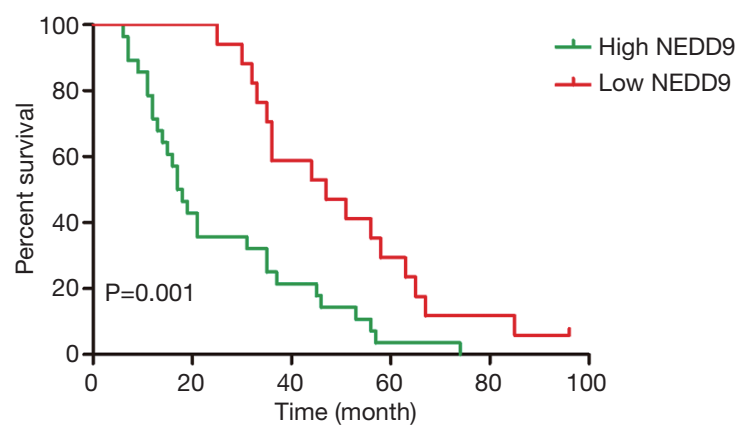

C

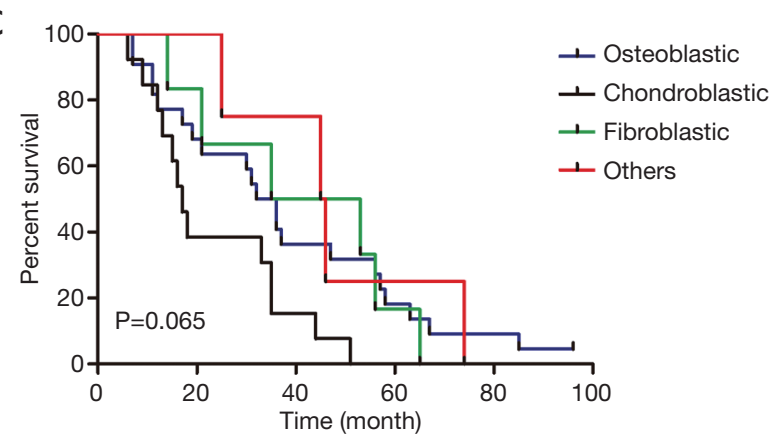

B

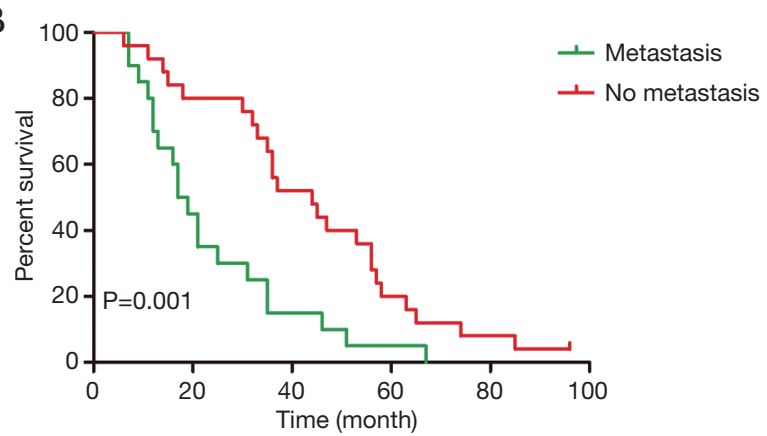

D

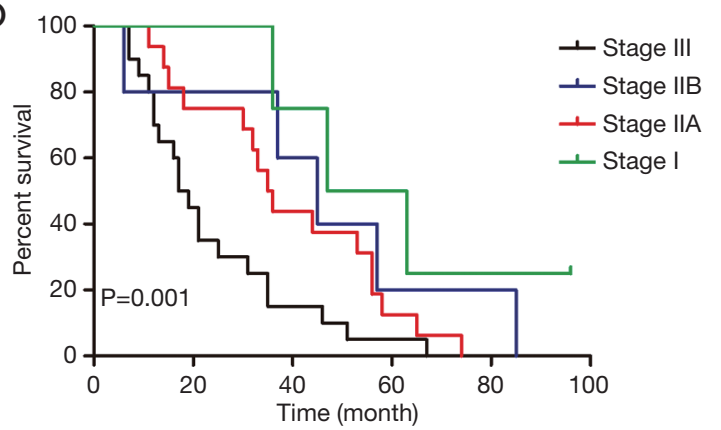

Figure 2 A Kaplan-Meier plot depicting overall survival of each osteosarcoma patient based on cancer NEDD9 expression (A, P=0.001), metastasis $(\mathrm{B}, \mathrm{P}=0.001)$, pathological types $(\mathrm{C}, \mathrm{P}=0.065)$, and Enneking stage $(\mathrm{D}, \mathrm{P}=0.001)$. $\mathrm{P}$ values were calculated by using log-rank analysis.

Table 2 The univariate and multivariate Cox regression analysis of osteosarcoma

\begin{tabular}{|c|c|c|c|c|c|c|}
\hline Characters & \multicolumn{3}{|c|}{ Univariate analysis } & \multicolumn{3}{|c|}{ Multivariate analysis } \\
\hline \multicolumn{7}{|l|}{ Sex } \\
\hline Female & 1 & & & & & \\
\hline Male & 1.65 & $0.89-3.06$ & 0.114 & & & \\
\hline$<20$ & 1 & & & & & \\
\hline$\geq 20$ & 1.06 & $0.53-2.11$ & 0.870 & & & \\
\hline \multicolumn{7}{|c|}{ Tumor size $(\mathrm{cm})$} \\
\hline$<8$ & 1 & & & & & \\
\hline Femur & 1 & & & & & \\
\hline Tibia & 1.03 & $0.51-2.07$ & 0.946 & & & \\
\hline Others & 1.42 & $0.64-3.14$ & 0.388 & & & \\
\hline
\end{tabular}

Table 2 (continued) 
Table 2 (continued)

\begin{tabular}{|c|c|c|c|c|c|c|}
\hline Characters & \multicolumn{3}{|c|}{ Univariate analysis } & \multicolumn{3}{|c|}{ Multivariate analysis } \\
\hline \multicolumn{7}{|l|}{ NEDD9 } \\
\hline Low-level & 1 & & & 1 & $2.51-14.4$ & $<0.001^{*}$ \\
\hline High-level & 2.79 & $1.46-5.34$ & $0.002^{*}$ & 6.03 & & \\
\hline \multicolumn{7}{|l|}{ Metastasis } \\
\hline No & 1 & & & 1 & $0.94-15.29$ & 0.061 \\
\hline Yes & 2.63 & $1.40-4.94$ & $0.003^{*}$ & 3.79 & & \\
\hline \multicolumn{7}{|l|}{ Stage } \\
\hline I & 1 & & & 1 & & \\
\hline III & 5.44 & $1.53-19.31$ & $0.009^{*}$ & 3.79 & $0.94-15.29$ & 0.061 \\
\hline \multicolumn{7}{|l|}{ Histopathology } \\
\hline Osteoblastic & 1 & & & 1 & & \\
\hline Chondroblastic & 2.59 & $1.21-5.51$ & $0.014^{*}$ & 1.65 & $0.72-3.77$ & 0.234 \\
\hline Fibroblastic & 1.04 & $0.41-2.60$ & 0.939 & 0.34 & $0.11-1.05$ & 0.060 \\
\hline Nonconventional & 0.82 & $0.28-2.41$ & 0.714 & 0.17 & $0.04-0.65$ & $0.010^{\star}$ \\
\hline
\end{tabular}

Statistical analyses were performed with Cox-regression. ${ }^{*}, \mathrm{P}<0.05$ was considered significant. HR, hazard ratio, Cl, confidence interval.

for osteosarcoma (22). Thus, it is necessary to find new prognostic biomarkers for osteosarcoma and develop new targeted drugs that prolong patient survival. A nonrandomized phase 2 trial involved using a combination of sorafenib and everolimus as a second-line treatment for advanced or unresectable osteosarcoma patients, showing that it did not reach the prespecified treatment target (23).

This research applied IHC as a method of examining NEDD9 expression levels in osteosarcoma tissue samples, indicating that NEDD9 is an independent prognostic biomarker for osteosarcoma, thereby increasing the possibility that future drug development for NEDD9 could focus on enhancing osteosarcoma therapy. Although NEDD9 has no existing chemical-specific inhibitors, microRNA such as miR-18a-5p or miR-5195-3p were shown to directly correlate with NEDD9 $(21,24)$. It is hoped that the findings of the present study will contribute to future studies on drug discovery and development in the specific biomarker treatment of osteosarcoma.

Certain limitations are present in the current study. First, selection bias may have affected the results, as the study involved data from one institution being retrospectively assessed. Therefore, additional verification should be conducted during large multi-center prospective cohort studies. Second, IHC was applied to assess NEDD9's clinical significance, yet was unable to solve inquiries involving explanations of processes.

\section{Conclusions}

According to the research outcomes, high NEDD9 expression present among human osteosarcoma tissue was significantly related to a poor OS rate. In addition, it is capable of identifying and making predictions about osteosarcoma. Future research could focus on developing a method that uses the NEDD9 expression level to identify high-risk patients and provide them with potential targeted treatment.

\section{Acknowledgments}

Funding: This study is supported by Startup Fund for 
Scientific Research, Fujian Medical University (Grant number: 2018QH2028).

\section{Footnote}

Reporting Checklist: The authors have completed the REMARK reporting checklist. Available at http://dx.doi. org/10.21037/tcr-20-1354a

Data Sharing Statement: Available at http://dx.doi. org/10.21037/tcr-20-1354a

Conflicts of Interest: All authors have completed the ICMJE uniform disclosure form (available at http://dx.doi. org/10.21037/tcr-20-1354a). The authors have no conflicts of interest to declare.

Ethical Statement: The authors are accountable for all aspects of the work in ensuring that questions related to the accuracy or integrity of any part of the work are appropriately investigated and resolved. The study was conducted in accordance with the Declaration of Helsinki (as revised in 2013). The study was approved by the Ethics Committees of the First Affiliated Hospital of Xiamen University (No.2019KY019) and all patients signed an informed consent form.

Open Access Statement: This is an Open Access article distributed in accordance with the Creative Commons Attribution-NonCommercial-NoDerivs 4.0 International License (CC BY-NC-ND 4.0), which permits the noncommercial replication and distribution of the article with the strict proviso that no changes or edits are made and the original work is properly cited (including links to both the formal publication through the relevant DOI and the license). See: https://creativecommons.org/licenses/by-nc-nd/4.0/.

\section{References}

1. Xia P, Gao X, Shao L, et al. Down-regulation of RAC2 by small interfering RNA restrains the progression of osteosarcoma by suppressing the Wnt signaling pathway. Int J Biol Macromol 2019;137:1221-31.

2. Tian $\mathrm{H}, \mathrm{Zhou} \mathrm{T}$, Chen $\mathrm{H}$, et al. Bone morphogenetic protein-2 promotes osteosarcoma growth by promoting epithelial-mesenchymal transition (EMT) through the $\mathrm{Wnt} / \beta$-catenin signaling pathway. J Orthop Res 2019;37:1638-48.
3. Feng J, Lan R, Cai G, et al. Verification of TREX1 as a promising indicator of judging the prognosis of osteosarcoma. J Orthop Surg Res 2016;11:150.

4. Gu Y, Lu J, Chen C, et al. NEDD9 overexpression predicts poor prognosis in solid cancers: a meta-analysis. Onco Targets Ther 2019;12:4213-22.

5. Izumchenko E, Singh MK, Plotnikova OV, et al. NEDD9 promotes oncogenic signaling in mammary tumor development. Cancer Res 2009;69:7198-206.

6. Bradbury P, Bach CT, Paul A, et al. Src kinase determines the dynamic exchange of the docking protein NEDD9 (neural precursor cell expressed developmentally downregulated gene 9) at focal adhesions. J Biol Chem 2014;289:24792-800.

7. Zheng M, McKeown-Longo PJ. Regulation of HEF1 expression and phosphorylation by TGF-beta 1 and cell adhesion. J Biol Chem 2002;277:39599-608.

8. Xu YF, Ge FJ, Han B, et al. High-mobility group box 1 expression and lymph node metastasis in intrahepatic cholangiocarcinoma. World J Gastroenterol 2015;21:3256-65.

9. Li P, Sun T, Yuan Q, et al. The expressions of NEDD9 and E-cadherin correlate with metastasis and poor prognosis in triple-negative breast cancer patients. Onco Targets Ther 2016;9:5751-9.

10. Lu P, Wang ZP, Dang Z, et al. Expression of NEDD9 in hepatocellular carcinoma and its clinical significance. Oncol Rep 2015;33:2375-83.

11. Bacci G, Ferrari S, Delepine N, et al. Predictive factors of histologic response to primary chemotherapy in osteosarcoma of the extremity: study of 272 patients preoperatively treated with high-dose methotrexate, doxorubicin, and cisplatin. J Clin Oncol 1998;16:658-63.

12. Zhao C, Li H, Wang L, et al. An Immunohistochemical Study of Stathmin 1 Expression in Osteosarcoma Shows an Association with Metastases and Poor Patient Prognosis. Med Sci Monit 2018;24:6070-8.

13. Huang Y, Liu W, He B, et al. Exosomes derived from bone marrow mesenchymal stem cells promote osteosarcoma development by activating oncogenic autophagy. J Bone Oncol 2020;21:100280.

14. Gabbasov R, Xiao F, Howe CG, et al. NEDD9 promotes oncogenic signaling, a stem/mesenchymal gene signature, and aggressive ovarian cancer growth in mice. Oncogene 2018;37:4854-70.

15. Kondo $S$, Iwata $S$, Yamada $T$, et al. Impact of the integrin signaling adaptor protein NEDD9 on prognosis and metastatic behavior of human lung cancer. Clin Cancer 
Res 2012;18:6326-38.

16. Miao Y, Li AL, Wang L, et al. Overexpression of NEDD9 is associated with altered expression of $\mathrm{E}$-Cadherin, $\beta$-Catenin and $\mathrm{N}$-Cadherin and predictive of poor prognosis in non-small cell lung cancer. Pathol Oncol Res 2013;19:281-6.

17. Ostojić J, Brčić L, Hrabač P, et al. Expression of Nedd9 in Transbronchial Biopsies of Lung Adenocarcinoma. Acta Clin Croat 2018;57:251-6.

18. Shagisultanova E, Gaponova AV, Gabbasov R, et al. Preclinical and clinical studies of the NEDD9 scaffold protein in cancer and other diseases. Gene 2015;567:1-11.

19. Zhao S, Min P, Liu L, et al. NEDD9 Facilitates HypoxiaInduced Gastric Cancer Cell Migration via MICAL1 Related Rac1 Activation. Front Pharmacol 2019;10:291.

20. McLaughlin SL, Ice RJ, Rajulapati A, et al. NEDD9 depletion leads to MMP14 inactivation by TIMP2 and prevents invasion and metastasis. Mol Cancer Res

Cite this article as: Feng J, Gu Y, Zhou L, Rui G, Liu W. High neural precursor cell-expressed developmentally downregulated 9 expression is a poor prognostic indicator for osteosarcoma. Transl Cancer Res 2020;9(11):7196-7204. doi: $10.21037 /$ tcr-20-1354a
2014;12:69-81.

21. Wang L, Shi G, Zhu D, et al. miR-5195-3p Suppresses Cell Proliferation and Induces Apoptosis by Directly Targeting NEDD9 in Osteosarcoma. Cancer Biother Radiopharm 2019;34:405-12.

22. Biermann JS, Chow W, Reed DR, et al. NCCN Guidelines Insights: Bone Cancer, Version 2.2017. J Natl Compr Canc Netw 2017;15:155-67.

23. Grignani G, Palmerini E, Ferraresi V, et al. Sorafenib and everolimus for patients with unresectable highgrade osteosarcoma progressing after standard treatment: a non-randomised phase 2 clinical trial. Lancet Oncol 2015;16:98-107.

24. Zheng Y, Nie P, Xu S. Long noncoding RNA linc00467 plays an oncogenic role in hepatocellular carcinoma by regulating the miR-18a-5p/NEDD9 axis. J Cell Biochem 2020;121:3135-44. 